Poincare Journal of Analysis E Applications

Vol. 2014 (1), Pages 39-46

CPoincare Publishers

\title{
EXACT SOLUTIONS FOR WICK-TYPE STOCHASTIC SAWADA-KOTERA EQUATION
}

\author{
HOSSAM A. GHANY ${ }^{\dagger}$ AND ABD-ALLAH HYDER
}

\author{
Date of Receiving : : May 14, 2014 \\ Date of Revision : June 14, 2014 \\ Date of Acceptance : June 27, 2014
}

\begin{abstract}
The variable coefficients Sawada-Kotera equations and Wick-type stochastic Sawada-Kotera equations are investigated. White noise functional solutions are shown by using the Hermite transform, homogeneous balance principle and the modified tanhcoth method. Moreover, some examples are given for the investigated model.
\end{abstract}

\section{Introduction}

It is well known that the solitons are stable against mutual collisions and behave like particles. In this sense, it is very important to study the nonlinear equations in random environment. However, variable coefficients nonlinear equations, as well as constant coefficients equations, cannot describe the realistic physical phenomena exactly. M. Wadati [17] first answered the interesting question. How does external noise affect the motion of solitons? and studied the diffusion of soliton of the KdV equation under Gaussian noise, which satisfies a diffusion equation in transformed coordinates. Wadati and Akutsu also studied the behaviors of solitons under the Gaussian white noise of the stochastic KdV equations with and without damping [19]. In addition, a nonlinear partial differential equation which describes wave propagations in random media was presented by Wadati [18]. Now, many researchers pay more attention to the study of the random waves, which are important subjects of stochastic partial differential equations, a number of soliton solutions of nonlinear stochastic partial differential equations and nonlinear stochastic fractional partial differential equations are obtained $[1,2,3,4,5,6,7,8,9,10,11,12,13,14,29,30,31]$. Holden et al.[11] researched stochastic partial differential equations in Wick versions via white noise functional

Key words and phrases. Sawada-Kotera equation; Hermite transform; Modified tanh-coth method; Wick- type; White noise.

The authors would like to thank the referees for reading carefully this paper and for useful suggestions.

PACS No.: 05.40.土a, 02.30.Jr.

Communicated by. S.K. Kaushik

${ }^{\dagger}$ Corresponding author. 